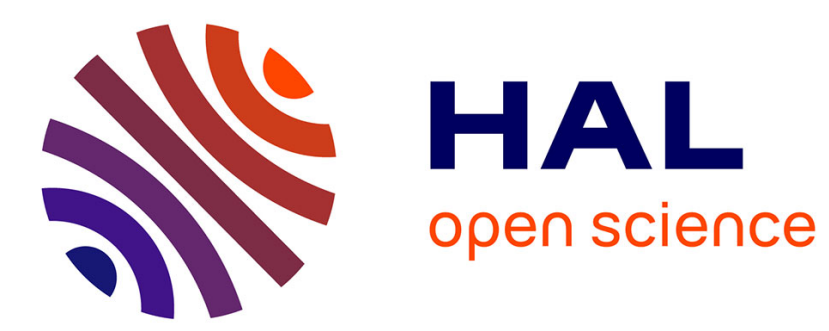

\title{
Some existence results for a quasilinear problem with source term in Zygmund-space
}

Boussad Hamour

\section{To cite this version:}

Boussad Hamour. Some existence results for a quasilinear problem with source term in Zygmundspace. Portugaliae Mathematica, 2020, 76 (3-4), pp.259 - 286. 10.4171/PM/2035 . hal-02900280

\section{HAL Id: hal-02900280 \\ https://hal.science/hal-02900280}

Submitted on 23 Jul 2020

HAL is a multi-disciplinary open access archive for the deposit and dissemination of scientific research documents, whether they are published or not. The documents may come from teaching and research institutions in France or abroad, or from public or private research centers.
L'archive ouverte pluridisciplinaire HAL, est destinée au dépôt et à la diffusion de documents scientifiques de niveau recherche, publiés ou non, émanant des établissements d'enseignement et de recherche français ou étrangers, des laboratoires publics ou privés.

$$
\text { Copyright }
$$




\title{
Some existence results for a quasilinear problem with source term in Zygmund-space
}

\author{
Boussad Hamour
}

Abstract. In this paper we study the existence of solution to the problem

$$
\left\{\begin{array}{l}
u \in H_{0}^{1}(\Omega), \\
-\operatorname{div}(A(x) D u)=H(x, u, D u)+f(x)+a_{0}(x) u \quad \text { in } \quad \mathcal{D}^{\prime}(\Omega),
\end{array}\right.
$$

where $\Omega$ is an open bounded set of $\mathbb{R}^{2}, A(x)$ a coercive matrix with coefficients in $L^{\infty}(\Omega)$, $H(x, s, \xi)$ a Carathéodory function satisfying, for some $\gamma>0$,

$$
-c_{0} A(x) \xi \xi \leq H(x, s, \xi) \operatorname{sign}(s) \leq \gamma A(x) \xi \xi \quad \text { a.e. } x \in \Omega, \quad \forall s \in \mathbb{R}, \quad \forall \xi \in \mathbb{R}^{2} .
$$

Here $f$ belongs to $L^{1}\left(\log L^{1}\right)(\Omega)$ and $a_{0} \geq 0$ to $L^{q}(\Omega), q>1$. For $f$ and $a_{0}$ sufficiently small, we prove the existence of at least one solution $u$ of this problem which is such that $e^{\delta_{0}|u|}-1$ belongs to $H_{0}^{1}(\Omega)$ for some $\delta_{0} \geq \gamma$ and satisfies an a priori estimate.

Keywords. Quasilinear problems; Existence; Zygmund-space.

\section{Introduction}

In this paper, we consider the quasilinear problem

$$
\left\{\begin{array}{l}
u \in H_{0}^{1}(\Omega) \\
-\operatorname{div}(A(x) D u)=H(x, u, D u)+f(x)+a_{0}(x) u \quad \text { in } \quad \mathcal{D}^{\prime}(\Omega),
\end{array}\right.
$$

where $\Omega$ is a bounded open subset of $\mathbb{R}^{2}, A$ a coercive matrix with bounded measurable coefficients, $H(x, s, \xi)$ a Carathéodory function having quadratic growth in $\xi$, more precisely, for some $\gamma>0$ and $c_{0} \geq 0$, one has

$$
\begin{aligned}
-c_{0} A(x) \xi \cdot \xi \leq H(x, s, \xi) \operatorname{sign}(s) \leq & \gamma A(x) \xi \cdot \xi \\
& \text { a.e. } x \in \Omega, \forall s \in \mathbb{R}, \quad \forall \xi \in \mathbb{R}^{2},
\end{aligned}
$$

where

$$
f \in L^{1}\left(\log L^{1}\right)(\Omega), f \neq 0, \quad \text { and } \quad 0 \leq a_{0} \in L^{q}(\Omega), q>1 \quad \text { with } \quad a_{0}>\neq 0 . \quad \text { (1.3) }
$$


For $f$ and $a_{0}$ are sufficiently small, in the sense that $f$ and $a_{0}$ satisfy the two smallness conditions (3.7) and (3.8), we prove that problem (1.1) has at least one solution, which is such that

$$
e^{\delta_{0}|u|}-1 \in H_{0}^{1}(\Omega),
$$

with

$$
\left\|\frac{e^{\delta_{0}|u|}-1}{\delta_{0}}\right\|_{H_{0}^{1}(\Omega)} \leq Z_{\delta_{0}}
$$

where $\delta_{0} \geq \gamma$ and $Z_{\delta_{0}}$ are two constants (see (7.9), (7.10), and (7.11) for their definitions) depending only on the data of the problem.

The main originality of our result is the fact that we assume that $a_{0}$ satisfies (1.3), namely that $a_{0}$ is a nonnegative function and $f \in L^{1}\left(\log L^{1}\right)(\Omega)$, with $f \neq 0$. The originality of this paper is twofold. First, we consider the case of source term $f$ in a class of Zygmund-space and no more in Lebesgue space [13]. Second, the fact that we assume that $a_{0}$ satisfies (1.3), namely that $a_{0}$ is a nonnegative function.

Let us begin with vast literature concerned with problems like (1.1) which has been studied in many papers in the case where $a_{0} \leq 0$.

Among these papers is a series of papers [8], [9], [10], [11] and [12] by L. Boccardo, F. Murat and J.-P. Puel (see also the paper [26] by J.-M. Rokotoson), which are concerned with the case where

$$
a_{0}(x) \leq-\alpha_{0}<0 .
$$

In these papers, the authors prove that when $a_{0}$ satisfies (1.6) and when $f$ belongs to $L^{q}(\Omega), q>\frac{N}{2}$, there exists at least one solution of (1.1) belonging to $L^{\infty}(\Omega)$ and satisfying some a priori estimates.

The case where

$$
a_{0}=0
$$

was considered, among others, by A. Alvino, P.-L. Lions and G. Trombetti in [1], by C. Maderna, C. Pagani and S. Salsa in [23], by V. Ferone and M.-R. Posteraro in [18], and by N. Grenon-Isselkou and J. Mossino in [19]. In these papers (which consider also nonlinear monotone operators), the authors prove that if $a_{0} \equiv 0$ and $f$ belonging to $L^{q}(\Omega), q>\frac{N}{2}$, with $\|f\|_{L^{q}(\Omega)}$ sufficiently small, there exists at least one $L^{\infty}(\Omega)$-solution of (1.1) which moreover belongs to $L^{\infty}(\Omega)$ and which satisfies some a priori estimates.

The case where $a_{0}$ satisfies (1.7) but $f$ belongs only to $L^{N / 2}(\Omega)$ for $N \geq 3$ (and no more to $L^{q}(\Omega)$ with $q>\frac{N}{2}$ ) was considered by V. Ferone and F. Murat in [15] (and in [16] in the nonlinear monotone case). These authors proved that when $\|f\|_{L^{N / 2}(\Omega)}$ is sufficiently small, there exists at least one solution of (1.1) such that 
$e^{\delta|u|}-1 \in H_{0}^{1}(\Omega)$ for some $\delta>\gamma$, and that such a solution satisfies an a priori estimate. Similar results were obtained in the case of nonlinear monotone operators where $f \in L^{1}(\log L)^{N-1}(\Omega)$ and $a_{0}$ satisfies (1.7) by F. Chiacchio in [13].

Finally, in [17], V. Ferone and F. Murat considered (also in the case of nonlinear monotone operators) the case where $a_{0}$ satisfies $a_{0} \leq 0$ and $f$ belongs to the Zygmund-space $L^{N / 2, \infty}(\Omega)$; in this case two smallness conditions should be fulfilled.

To finish with the case where $a_{0}$ satisfies $a_{0} \leq 0$ and $f$ belongs to $H^{-1}(\Omega)$, let us quote the papers [7] by L. Boccardo, F. Murat and J.-P. Puel and [8] by A. Bensoussan, L. Boccardo and F. Murat, where these authors proved the existence of at least one solution of (1.1).

In contrast with the cases (1.6) and (1.7), the present paper is concerned with the case (1.3) where $a_{0} \geq 0$, and $a_{0} \neq 0$. In this setting we are only aware of four recent papers. In [20], B. Hamour and F. Murat proved a similar result to the one of the present paper, for $\|f\|_{L^{N / 2}(\Omega)}$ and $\left\|a_{0}\right\|_{q}$ are sufficiently small, with $N \geq 3$ and $q>\frac{N}{2}$. In [22], L. Jeanjean and B. Sirakov proved too a result similar to the one of the present paper when $f \in L^{q}(\Omega), q>\frac{N}{2}$, they also proved the existence of at least two solutions of (1.1). In [3], D. Arcoya, C. De Coster, L. Jeanjean and $\mathrm{K}$. Tanaka proved the existence of a continuum $(u, \lambda)$ of solutions (with $u$ belonging moreover to $\left.L^{\infty}(\Omega)\right)$ when $A(x)=I d, H(x, s, \xi)=\mu(x)|\xi|^{2}$, with $\mu \in L^{\infty}(\Omega)$, $\mu(x) \geq \mu>0, f \in L^{q}(\Omega), q>\frac{N}{2}, f \geq 0, f \neq 0$ and $a_{0}(x)=\lambda a_{0}^{\star}(x)$ with $a_{0}^{\star} \in L^{q}(\Omega), a_{0}^{\star} \geq 0$ and $a_{0}^{\star} \neq 0$; moreover, under some further conditions on $f$, and that there are at least two nonnegative solutions of (1.1) when $\lambda>0$ is sufficiently small.

In [27], in a similar setting, assuming only that $\mu(x) \geq 0$ but that the supports of $\mu$ and of $a_{0}^{\star}$ have a nonempty intersection and that $N \leq 5$, P. Souplet proved the existence of a continuum $(u, \lambda)$ of solutions, and that there are at least two nonnegative solutions of (1.1) when $\lambda>0$ is sufficiently small.

With respect to the results obtained in the four latest papers, we prove in this paper, as said above, the existence of (only) one solution of (1.1) in the case $a_{0} \geq 0$ when $a_{0}$ and $f$ satisfy the two smallness conditions (3.7) and (3.8), but our result is obtained in the general case of a nonlinearity $H(x, s, \xi)$ which satisfies only (1.2), with $f \in L^{1}\left(\log L^{1}\right)(\Omega)$ and with $a_{0} \in L^{q}(\Omega), q>1$. 
The paper is organized as follows:

The (short) Section 2 deals with preliminaries results about Zygmund-spaces. The precise statement of our result is given in Section 3 (Theorem 3.1), as well as the precise assumptions under which we are able to prove it. These assumptions include in particular the two smallness conditions (3.7) and (3.8).

Our method for proving Theorem 3.1 is based on an equivalence result (see Theorem 4.2) stated in Section 4 once we have introduced the functions $K_{\delta}(x, s, \zeta)$ and $g_{\delta}(s)$ (see (4.5) and (4.6)) and made some technical remarks on them. This equivalence result is very close to that given in the paper [16] by V. Ferone and F. Murat.

Our equivalence result permuts us to reduce the proof of existence of a solution $u$ of (1.1), which satisfies (1.4) and (1.5), to the existence (see Theorem 4.3) of a function $w$ related to $u$ by

$$
w=\frac{1}{\delta_{0}}\left(e^{\delta_{0}|u|}-1\right) \operatorname{sign}(u),
$$

which solve the problem (see (4.13))

$$
\left\{\begin{array}{l}
w \in H_{0}^{1}(\Omega) \\
-\operatorname{div}(A(x) D w)+K_{\delta_{0}}(x, w, D w) \operatorname{sign}(w) \\
\quad=\left(1+\delta_{0}|w|\right) f(x)+a_{0}(x) w+a_{0}(x) g_{\delta_{0}}(w) \operatorname{sign}(w) \text { in } \mathcal{D}^{\prime}(\Omega),
\end{array}\right.
$$

and satisfies the estimate see (4.14)

$$
\|w\|_{H_{0}^{1}(\Omega)} \leq Z_{\delta_{0}}
$$

Our goal thus becomes to prove Theorem 4.3, namely to prove the existence of a solution $w$ which satisfies (4.13) and (4.14).

Problem (4.13) is very similar to Problem (1.1), since it involves a term $-K_{\delta_{0}}(x, w, D w) \operatorname{sign}(w)$ which has quadratic growth in $D w$, as well as a zeroth order term is given by $\delta_{0}|w| f(x)+a_{0}(x) w+a_{0}(x) g_{\delta_{0}}(w) \operatorname{sign}(w)$. But this problem is also very different from (1.1), since the term $-K_{\delta_{0}}(x, w, D w) \operatorname{sign}(w)$ with quadratic growth has the "good sign property", since $K_{\delta_{0}}(x, s, \xi)$ satisfies

$$
K_{\delta_{0}}(x, s, \xi) \geq 0
$$

while the zeroth order term is now no more a linear but a semilinear term with $|s|^{2}$ growth due to presence of the term $a_{0}(x) g_{\delta_{0}}(w) \operatorname{sign}(w)$.

We will prove Theorem 4.3 essentially by applying Schauder's fixed point theorem. But there are some difficulties to do it directly, since the term with quadratic growth $K_{\delta_{0}}(x, w, D w) \operatorname{sign}(w)$ only belongs to $L^{1}(\Omega)$ in general. We therefore begin by defining an approximate problem (see (5.1)) where $K_{\delta}(x, w, D w)$ is replaced by its truncation at height $k$, namely $T_{k}\left(K_{\delta}(x, w, D w)\right.$ ), and we prove (see Theorem 5.1) that if $f$ and $a_{0}$ satisfy the two smallness conditions (3.7) and (3.8), 
this approximate problem has at least one solution $w_{k}$ which satisfies the a priori estimate

$$
\left\|w_{k}\right\|_{H_{0}^{1}(\Omega)} \leq Z_{\delta_{0}} .
$$

This result is obtained in Section 5 by applying Schauder's fixed point theorem in a classical way.

We then pass to the limit as $k$ tends to infinity and we prove in Section 6 that (for a subsequence of $k$ ) $w_{k}$ tend to some $w^{\star}$ strongly in $H_{0}^{1}(\Omega)$ and that this $w^{\star}$ is a solution of (4.13) which also satisfies (4.14) (see End of the proof of Theorem 4.3).

This completes the proof of Theorem 4.3, and therefore proves Theorem 3.1, as announced.

This proof follows along the lines of the proof used by V. Ferone and F. Murat in [15] in the case where $a_{0}=0$. As mentionned above, this method can be applied to the nonlinear case where the linear operator $-\operatorname{div}(A(x) D u)$ is replaced by a Leray-Lions operator $-\operatorname{div}(a(x, u, D u))$ working in $W_{0}^{1, p}(\Omega)$ for some $1<p<N$ and $p=N$, and where the quasilinear term $H(x, u, D u)$ has $p$-growth in $|D u|$, as it was done in [16] by V. Ferone and F. Murat in this nonlinear setting when $a_{0}=0$. This will be the goal of our next paper [21].

\section{Preliminary result}

In this section we recall some definitions and classical properties about rearrangements and introduce the Zygmund-spaces. Let $\Omega$ be an open bounded subset of $\mathbb{R}^{N}$ equipped with the Lebesgue's measure and let $\phi: \Omega \rightarrow \mathbb{R}$ be a measurable function, we define the distribution function $\mu_{\phi}$ by

$$
\mu_{\phi}(t)=\operatorname{meas}\{x \in \Omega:|\phi(x)|>t\}, \quad \forall t \geq 0,
$$

and the decreasing rearrangement of $\phi$ by

$$
\phi^{*}(s)=\sup \left\{t>0: \mu_{\phi}(t)>s\right\}, \quad \forall s \in(0,|\Omega|) \quad(|\Omega|=\text { meas }(\Omega)) .
$$

We recall only the following Hardy-Littlewood inequality (see [24])

$$
\int_{\Omega}|g(x) h(x)| d x \leq \int_{0}^{|\Omega|} g^{*}(s) h^{*}(s) d s,
$$

for any $g$ and $h$ real measurable functions defined in $\Omega$.

The Zygmund-space $L^{p}(\log L)^{q}, p>0$ and $q \in \mathbb{R}$, consists of all measurable functions $\phi: \Omega \rightarrow \mathbb{R}$ such that the following quantity is finite.

$$
\|\phi\|_{L^{p}(\log L)^{q}}=\left(\int_{0}^{|\Omega|}\left(\left(\log \frac{|\Omega|}{s}\right)^{q} \phi^{*}(s)\right)^{p} d s\right)^{1 / p} .
$$


Finally, we recall the following Theorem (see [2]).

Theorem 2.1. If $u$ is a real function in $W_{0}^{1, N}(\Omega)$, then

$$
u^{*}(s) \leq \frac{\|D u\|_{N}}{N \omega_{N}^{1 / N}}\left(\log \frac{|\Omega|}{s}\right)^{\frac{N-1}{N}} \quad \forall s \in(0,|\Omega|),
$$

where $w_{N}$ is the the measure of unit sphere of $\mathbb{R}^{N}$ such that

$$
w_{N}=\frac{\pi^{N / 2}}{\Gamma(1+N / 2)} .
$$

In particular, for $N=2$ one has

$$
N \omega_{N}^{1 / N}=(2 \pi)^{1 / 2}
$$

\section{The main result}

In this paper we consider the following quasilinear problem

$$
\left\{\begin{array}{l}
u \in H_{0}^{1}(\Omega), \\
-\operatorname{div}(A(x) D u)=H(x, u, D u)+a_{0}(x) u+f(x) \text { in } \mathcal{D}^{\prime}(\Omega),
\end{array}\right.
$$

where $\Omega$ is bounded open subset of $\mathbb{R}^{2}$, where $A$ is a coercive matrix with bounded mesurable coefficients, i.e.,

$$
\left\{\begin{array}{l}
A \in\left(L^{\infty}(\Omega)\right)^{2 \times 2} \\
\exists \alpha>0, \quad A(x) \xi \xi \geq \alpha|\xi|^{2} \quad \text { a.e. } x \in \Omega, \quad \forall \xi \in \mathbb{R}^{2},
\end{array}\right.
$$

where $H: \Omega \times \mathbb{R} \times \mathbb{R}^{2} \rightarrow \mathbb{R}$ is a Carathéodory function which satisfies the condition:

$$
-c_{0} A(x) \xi \xi \leq H(x, s, \xi) \operatorname{sign}(s) \leq \gamma A(x) \xi \xi, \text { a.e. } x \in \Omega, \forall s \in \mathbb{R}, \forall \xi \in \mathbb{R}^{2},
$$

for a given $\gamma>0$ and $c_{0} \geq 0$, where the function $\operatorname{sign}(s)$ is defined by:

$$
\operatorname{sign}(s)=\left\{\begin{array}{cl}
+1 & \text { if } \quad s>0 \\
0 & \text { if } \quad s=0 \\
-1 & \text { if } \quad s<0
\end{array}\right.
$$

where the coefficient $a_{0}$ satisfies

$$
a_{0} \in L^{q}(\Omega) \text { for some } q>1, \quad a_{0} \geq 0, \quad a_{0} \neq 0,
$$


and finally where

$$
f \in L^{1}\left(\log L^{1}\right)(\Omega), \quad f \neq 0 .
$$

Since $\Omega$ is bounded, we equip the space $H_{0}^{1}(\Omega)$ with the gradient norm

$$
\|u\|_{H_{0}^{1}(\Omega)}=\|D u\|_{L^{2}(\Omega)^{2}} .
$$

We finally assume that $f$ and $a_{0}$ are sufficiently small, and more precisely that

$$
\begin{gathered}
\alpha-C_{1}(q)\left\|a_{0}\right\|_{q}-\gamma\|f\|_{L^{1}(\log L)(\Omega)}>0, \\
\|f\|_{H^{-1}(\Omega)} \leq \frac{\left(2 \pi \alpha-C_{1}(q)\left\|a_{0}\right\|_{q}-\delta\|f\|_{L^{1}(\log L)(\Omega)}\right)^{2}}{16 \pi^{2} \delta_{1} C_{2}(q)\left\|a_{0}\right\|_{q}},
\end{gathered}
$$

where the constant $\delta_{1}$ is defined by (7.5) and where some constants $C_{1}(q)>0$ and $C_{2}(q)>0$, as well as

$$
C_{1}(q)=\left(\int_{0}^{|\Omega|}\left(\log \frac{|\Omega|}{s}\right)^{q^{\prime}} d s\right)^{1 / q^{\prime}}, \quad C_{2}(q)=\left(\int_{0}^{|\Omega|}\left(\log \frac{|\Omega|}{s}\right)^{\frac{3 q^{\prime}}{2}} d s\right)^{1 / q^{\prime}}
$$

where $q^{\prime}$ the Hölder's conjugate of the exponent $q$, i.e.

$$
\frac{1}{q}+\frac{1}{q^{\prime}}=1
$$

Our aim is to prove the following Theorem:

Theorem 3.1. Assume that (3.2), (3.3), (3.4), (3.5), (3.6) hold true. Assume moreover that the two smallness conditions (3.7) and (3.8) hold true.

Then there exist a constant $\delta_{0}$ with $\delta_{0} \geq \gamma$ and a constant $Z_{\delta_{0}}$, such that there exists at least one solution $u$ of (3.1) which further satisfies

$$
\left(e^{\delta_{0}|u|}-1\right) \in H_{0}^{1}(\Omega),
$$

with

$$
\left\|e^{\delta_{0}|u|} D u\right\|_{L^{2}(\Omega)^{2}}=\left\|\frac{e^{\delta_{0}|u|}-1}{\delta_{0}}\right\|_{H_{0}^{1}(\Omega)} \leq Z_{\delta_{0}} .
$$

Remark 3.2. The definitions of the constants $\delta_{0}$ and $Z_{\delta_{0}}$ which appear in Theorem 3.1 are given in Appendix 7 (see Lemma 7.2). These definitions are based on the properties of the family of functions $\phi_{\delta}$ which look like convex parabolas (see Fig. 2). The constant $\delta_{0}$ is the unique value of the parameter $\delta_{0}$ for which the function $\phi_{\delta_{0}}$ admits to double zero and $Z_{\delta_{0}}$ is the value such that $\phi_{\delta}\left(Z_{\delta_{0}}\right)=0$.

\section{An equivalence result}

The main results of this Section are Theorems 4.2 and 4.3 .

Indeed, as said above, the proof of Theorem 3.1 is based on the equivalence result of Theorem 4.2 that we state and we prove in this section. Here we always assume that 
$\delta>0$ and let us first proceed with a formal computation. If $u$ is a solution of the following problem:

$$
\left\{\begin{array}{l}
-\operatorname{div}(A(x) D u)=H(x, u, D u)+f(x)+a_{0}(x) u \text { in } \Omega, \\
u=0 \text { on } \partial \Omega,
\end{array}\right.
$$

and if we formally define the function $w_{\delta}$ by

$$
w_{\delta}=\frac{1}{\delta}\left(e^{\delta|u|}-1\right) \operatorname{sign}(u),
$$

where the function sign is defined by (3.4), we have, at least formally,

$$
\left\{\begin{array}{l}
e^{\delta|u|}=1+\delta\left|w_{\delta}\right|, \quad|u|=\frac{1}{\delta} \log \left(1+\delta\left|w_{\delta}\right|\right), \quad \operatorname{sign}(u)=\operatorname{sign}\left(w_{\delta}\right), \\
D w_{\delta}=e^{\delta|u|} D u, \\
-\operatorname{div}\left(A(x) D w_{\delta}\right)=-\delta e^{\delta|u|} A(x) D u D u \operatorname{sign}(u)-e^{\delta|u|}(\operatorname{div}(A(x) D u)),
\end{array}\right.
$$

and therefore $w_{\delta}$ is, at least formally, a solution of

$$
\left\{\begin{aligned}
-\operatorname{div}\left(A(x) D w_{\delta}\right)= & -K_{\delta}\left(x, w_{\delta}, D w_{\delta}\right) \operatorname{sign}\left(w_{\delta}\right) \\
& +\left(1+\delta\left|w_{\delta}\right|\right) f(x)+a_{0}(x) w_{\delta} \\
& +a_{0}(x) g_{\delta}\left(w_{\delta}\right) \operatorname{sign}\left(w_{\delta}\right) \text { in } \Omega, \\
w_{\delta}=0 \text { on } \partial \Omega, &
\end{aligned}\right.
$$

where the functions $K_{\delta}: \Omega \times \mathbb{R} \times \mathbb{R}^{N} \rightarrow \mathbb{R}$ and $g_{\delta}: \mathbb{R} \rightarrow \mathbb{R}$ are defined by the following formulas:

$$
\left\{\begin{array}{l}
K_{\delta}(x, t, \zeta)= \\
=\frac{\delta}{1+\delta|t|} A(x) \zeta \zeta-(1+\delta|t|) H\left(x, \frac{1}{\delta} \log (1+\delta|t|) \operatorname{sign}(t), \frac{\zeta}{1+\delta|t|}\right) \operatorname{sign}(t) \\
\text { a.e. } x \in \Omega, \quad \forall t \in \mathbb{R}, \quad \forall \zeta \in \mathbb{R}^{N}
\end{array}\right.
$$

and

$$
g_{\delta}(t)=-|t|+\frac{1}{\delta}(1+\delta|t|) \log (1+\delta|t|), \quad \forall t \in \mathbb{R} .
$$

Conversely, if $w_{\delta}$ is a solution of (4.4), and if we formally define the function by

$$
u=\frac{1}{\delta} \log \left(1+\delta\left|w_{\delta}\right|\right) \operatorname{sign}\left(w_{\delta}\right)
$$

the some formal computation easily shows that $u$ is solution of 4.1.

Remark 4.1. Observe that the functions $K_{\delta}(x, w, D w)$ and $K_{\delta}(x, w, D w) \operatorname{sign}(w)$ are correctly defined and are mesurable functions when $w \in H^{1}(\Omega)$, and continuous with respect to the almost everywhere convergence of $w$ and $D w$ (see Lemma 3.3 in [20]) 
Theorem 4.2. Assume that (3.2), (3.3), (3.4), (3.5), (3.6) hold true, and let $\delta>0$ be fixed. Let the functions $K_{\delta}$ and $g_{\delta}$ be defined by (4.5) and (4.6).

If $u$ is any solution of (3.1) which satisfies

$$
\left(e^{\delta|u|}-1\right) \in H_{0}^{1}(\Omega)
$$

then the function $w_{\delta}$ defined by (4.2), namely by

$$
w_{\delta}=\frac{1}{\delta}\left(e^{\delta|u|}-1\right) \operatorname{sign}(u)
$$

satisfies

$$
\left\{\begin{array}{l}
w_{\delta} \in H_{0}^{1}(\Omega) \\
-\operatorname{div}\left(A(x) D w_{\delta}\right)+K_{\delta}\left(x, w_{\delta}, D w_{\delta}\right) \operatorname{sign}\left(w_{\delta}\right) \\
=\left(1+\delta\left|w_{\delta}\right|\right) f(x)+a_{0}(x) w_{\delta}+a_{0}(x) g_{\delta}\left(w_{\delta}\right) \operatorname{sign}\left(w_{\delta}\right) \text { in } \mathcal{D}^{\prime}(\Omega)
\end{array}\right.
$$

Conversely, if $w_{\delta}$ is any solution of (4.9), then the function $u$ defined by

$$
u=\frac{1}{\delta} \log \left(1+\delta\left|w_{\delta}\right|\right) \operatorname{sign}\left(w_{\delta}\right)
$$

is a solution of (3.1) which satisfies (4.8).

Proof of Theorem 4.2. Define the function $\hat{f}$ by

$$
\hat{f}(x)=f(x)+a_{0}(x) u(x)
$$

In view of (4.2) and of the definition (4.6) of $g_{\delta}(s)$, one has

$$
\left\{\begin{array}{l}
\left(1+\delta\left|w_{\delta}\right|\right) f(x)+a_{0}(x) w_{\delta}+a_{0}(x) g_{\delta}\left(w_{\delta}\right) \operatorname{sign}\left(w_{\delta}\right) \\
=\left(1+\delta\left|w_{\delta}\right|\right)\left(f(x)+a_{0}(x) \frac{1}{\delta} \log \left(1+\delta\left|w_{\delta}\right|\right) \operatorname{sign}\left(w_{\delta}\right)\right) \\
=\left(1+\delta\left|w_{\delta}\right|\right)\left(f(x)+a_{0}(x) u(x)\right)=\left(1+\delta\left|w_{\delta}\right|\right) \hat{f}(x)
\end{array}\right.
$$

Then Theorem 4.2 becomes an immediate application of Proposition 1.8 of [15], once one observes that

$$
\hat{f} \in L^{1}(\Omega)
$$

Indeed $a_{0} u \in L^{1}(\Omega)$, since $a_{0}$ is assumed to belong to $L^{q}(\Omega), q>1$, while $u \in L^{r}(\Omega)$ for every $r<\infty$, since $\left(e^{\delta|u|}-1\right) \in H_{0}^{1}(\Omega)$, hence in particular to $L^{1}(\Omega)$, which implies that $e^{\delta|u|}$ belongs to $L^{1}(\Omega)$. Theorem 4.2 is therefore proved.

From the equivalence Theorem 4.2 one immediately deduces that Theorem 3.1 is equivalent to the following Theorem. 
Theorem 4.3. Assume that (3.2), (3.3), (3.4), (3.5), (3.6) hold true. Assume moreover that the two smallness conditions (3.7) and (3.8) hold true.

Then there exist a constant $\delta_{0}$ with $\delta_{0} \geq \gamma$, and a constant $Z_{\delta_{0}}$, such that there exists at least one solution $w$ of

$$
\left\{\begin{array}{l}
w \in H_{0}^{1}(\Omega), \\
-\operatorname{div}(A(x) D w)+K_{\delta_{0}}(x, w, D w) \operatorname{sign}(w) \\
=\left(1+\delta_{0}|w|\right) f(x)+a_{0}(x) w+a_{0}(x) g_{\delta_{0}}(w) \operatorname{sign}(w) \text { in } \mathcal{D}^{\prime}(\Omega),
\end{array}\right.
$$

which satisfies

$$
\|w\|_{H_{0}^{1}(\Omega)} \leq Z_{\delta_{0}}
$$

The rest of this paper will therefore be devoted to the proof of Theorem 4.3. This will be done in two steps: first, we will prove the existence of a solution for an approximate problem; second, we will pass to the limit in this approximate problem.

Remark 4.4. We assume that (3.2), (3.3), (3.5), (3.6) hold true. Assume moreover that the two smallness conditions (3.7) and (3.8) hold true, we try to explain how these two conditions come from an "a priori estimate" that one can obtain on the solutions of (4.9).

If $w_{\delta}$ is any solution of (4.9), using $T_{k}\left(w_{\delta}\right) \in H_{0}^{1}(\Omega) \cap L^{\infty}(\Omega)$ as test function, where $T_{k}: \mathbb{R} \rightarrow \mathbb{R}$ is the usual truncation at height $k$ defined by

$$
T_{k}(s)=\left\{\begin{aligned}
-k & \text { if } \quad s \leq-k \\
s & \text { if } \quad-k \leq s \leq+k \\
+k & \text { if } \quad+k \leq s
\end{aligned}\right.
$$

one has

$$
\left\{\begin{aligned}
\int_{\Omega} & A(x) D w_{\delta} D T_{k}\left(w_{\delta}\right) d x+\int_{\Omega} K_{\delta}\left(x, w_{\delta}, D w_{\delta}\right)\left|T_{k}\left(w_{\delta}\right)\right| d x \\
= & \int_{\Omega} f(x) T_{k}\left(w_{\delta}\right) d x+\int_{\Omega} \delta f(x)\left|w_{\delta}\right| T_{k}\left(w_{\delta}\right) d x \\
& +\int_{\Omega} a_{0}(x) w_{\delta} T_{k}\left(w_{\delta}\right) d x+\int_{\Omega} a_{0}(x) g_{\delta}\left(w_{\delta}\right)\left|T_{k}\left(w_{\delta}\right)\right| d x .
\end{aligned}\right.
$$

We assume that $\delta$ satisfies

$$
\gamma \leq \delta \leq \delta_{1}
$$

where $\delta_{1}$ is defined by (7.5).

Since $\delta \geq \gamma$ by (4.17), we deduce that $K_{\delta}(x, s, \zeta) \geq 0$ (see Remark 3.1 in [20]). Using this fact and passing to the limit as $k$ tends to $+\infty$, we obtain

$$
\left\{\begin{aligned}
\int_{\Omega} A(x) D w_{\delta} D w_{\delta} d x & \leq \int_{\Omega} f(x) w_{\delta} d x+\int_{\Omega} \delta f(x)\left|w_{\delta}\right|^{2} d x \\
& +\int_{\Omega} a_{0}(x)\left|w_{\delta}\right|^{2} d x+\int_{\Omega} a_{0}(x) g_{\delta}\left(w_{\delta}\right)\left|w_{\delta}\right| d x .
\end{aligned}\right.
$$

In (4.18) we use the left-hand side the fact that the matrix is coercive, in all terms of the right-hand side the fact that $f \in L^{1}(\log L)(\Omega)$, Holder's inequality, Hardy-Littlewood's 
inequality, classical properties about rearrangements and the fact

$$
0 \leq g_{\delta}(t) \leq \delta|t|^{2}, \quad \forall t \in \mathbb{R}, \quad t \neq 0, \quad \forall \delta, \quad 0<\delta \leq \delta_{1} .
$$

This allows us to obtain an estimate on $T_{k}\left(w_{\delta}\right)$, in which we pass to the limit in $k$ to get

$$
\left\{\begin{array}{c}
\alpha\left\|D w_{\delta}\right\|_{2}^{2}<\|f\|_{H^{-1}(\Omega)}\left\|D w_{\delta}\right\|_{2}+\frac{\delta\|f\|_{L^{1}(\log L)(\Omega)}}{2 \pi}\left\|D w_{\delta}\right\|_{2}^{2} \\
+\frac{C_{1}(q)\left\|a_{0}\right\|_{q}}{2 \pi}\left\|D w_{\delta}\right\|_{2}^{2}+\frac{\delta C_{2}(q)\left\|a_{0}\right\|_{q}}{(2 \pi)^{3 / 2}} \mid D w_{\delta} \|_{2}^{3} \quad \text { if } w_{\delta} \neq 0
\end{array}\right.
$$

where these constants $C_{1}(q)$ and $C_{2}(q)$ depend only on $q$ (see (3.9)).

Dividing by $\left\|D w_{\delta}\right\|_{2}$ this implies that

$$
\left\{\begin{array}{l}
\alpha\left\|D w_{\delta}\right\|_{2}<\|f\|_{H^{-1}(\Omega)}+\frac{\delta\|f\|_{L^{1}(\log L)(\Omega)}}{2 \pi}\left\|D w_{\delta}\right\|_{2} \\
+\frac{C_{1}(q)\left\|a_{0}\right\|_{q}}{2 \pi}\left\|D w_{\delta}\right\|_{2}+\frac{\delta C_{2}(q)\left\|a_{0}\right\|_{q}}{(2 \pi)^{3 / 2}} \mid D w_{\delta} \|_{2}^{2} \quad \text { if } w_{\delta} \neq 0
\end{array}\right.
$$

Using (4.17) in the fourth term of the right-hand side of (4.20), we have

$$
\left\{\begin{array}{l}
\alpha\left\|D w_{\delta}\right\|_{2}<\|f\|_{H^{-1}(\Omega)}+\frac{\delta\|f\|_{L^{1}(\log L)(\Omega)}}{2 \pi}\left\|D w_{\delta}\right\|_{2} \\
+\frac{C_{1}(q)\left\|a_{0}\right\|_{q}}{2 \pi}\left\|D w_{\delta}\right\|_{2}+\frac{\delta_{1} C_{2}(q)\left\|a_{0}\right\|_{q}}{(2 \pi)^{3 / 2}} \mid D w_{\delta} \|_{2}^{2} \quad \text { if } w_{\delta} \neq 0,
\end{array}\right.
$$

In view of the definition (7.7) of the function $\Phi_{\delta}$ (see also Fig. 2), we have proved that if $w_{\delta}$ is any solution of (4.9), one has

$$
\Phi_{\delta}\left(\left\|D w_{\delta}\right\|_{2}\right)>0 \quad \text { if } \quad \gamma \leq \delta \leq \delta_{1} .
$$

But by the definition of $\delta_{0}$, one has

$$
\Phi_{\delta}(X)>0, \quad \forall X \geq 0, \quad \forall \delta, 0<\delta \leq \delta_{1},
$$

and therefore inequality (4.22) does not imply anything on $\left\|D w_{\delta}\right\|_{2}$ when $\delta$ satisfies $\delta_{0}<\delta \leq \delta_{1}$. In contrast, when $\delta<\delta_{0}$, the strict inequality (4.22) implies that

$$
\text { either }\left\|D w_{\delta}\right\|_{2}<Y_{\delta}^{-} \quad \text { or }\left\|D w_{\delta}\right\|_{2}>Y_{\delta}^{+} \quad \text { if } \delta<\delta_{0},
$$

where $Y_{\delta}^{-}<Y_{\delta}^{+}$are the two distinct zeros of the function $\Phi_{\delta}$ (see Remarks 7.3 and Fig. 2 ), while when $\delta=\delta_{0}$, the strict inequality (4.22) implies that

$$
\text { either }\left\|D w_{\delta_{0}}\right\|_{2}<Z_{\delta_{0}} \quad \text { or }\left\|D w_{\delta_{0}}\right\|_{2}>Z_{\delta_{0}} \quad \text { if } \delta=\delta_{0} .
$$

Inequalities (4.23) and (4.24) are not a priori estimates, since they do not imply any bound on $\left\|D w_{\delta}\right\|_{2}$. Nevertheless these inequalities exclude the closed interval $\left[Y_{\delta}^{-}, Y_{\delta}^{+}\right]$ or the point $Z_{\delta_{0}}$ for $\left\|D w_{\delta}\right\|_{2}$, and they give the hope to prove the existence of a fixed point in the set $\left\|D w_{\delta}\right\|_{2} \leq Y_{\delta}^{-}$, when $\delta<\delta_{0}$, or in the set $\left\|D w_{\delta_{0}}\right\|_{2} \leq Z_{\delta_{0}}$, when $\delta=\delta_{0}$.

These inequalities also explain where the two smallness conditions (3.7) and (3.8) come from. Indeed (see Remark 7.3), these two smallness conditions imply that the value $\delta_{0}$ of $\delta$ for which $\Phi_{\delta}$ has a double zero satisfies $\delta_{0} \geq \gamma$, which is the case where, as said just above, some hope is allowed. 


\section{Existence of a solution for an approximate problem}

In this Section we introduce an approximation of problem (4.13). Under the two smallness conditions (3.7) and (3.8), we prove by applying Schauder's fixed point theorem that this approximate problem has at least one solution which satisfies the estimate (4.14).

Let $\delta_{0}$ be defined by (7.9), (7.10) and (7.11). For any $k>0$, we consider the following approximate problem.

$$
\left\{\begin{array}{l}
w_{k} \in H_{0}^{1}(\Omega) \\
-\operatorname{div}\left(A(x) D w_{k}\right)+T_{k}\left(K_{\delta_{0}}\left(x, w_{k}, D w_{k}\right)\right) \operatorname{sign}_{k}\left(w_{k}\right) \\
=\left(1+\delta_{0}\left|w_{k}\right|\right) f(x)+a_{0}(x) w_{k}+a_{0}(x) g_{\delta_{0}}\left(w_{k}\right) \operatorname{sign}\left(w_{k}\right) \text { in } \mathcal{D}^{\prime}(\Omega),
\end{array}\right.
$$

where $T_{k}$ is the usual truncation at height $k$ defined by (4.15) and where $\operatorname{sign}_{k}: \mathbb{R} \rightarrow \mathbb{R}$ is the approximation of the function sign which is defined by

$$
\operatorname{sign}_{k}(s)= \begin{cases}k s & \text { if } \quad|s| \leq \frac{1}{k} \\ \operatorname{sign}(s) & \text { if } \quad|s| \geq \frac{1}{k} .\end{cases}
$$

Theorem 5.1. Assume that (3.2), (3.3), (3.5) and (3.6) hold true. Assume moreover that the two smallness conditions (3.7) and (3.8) hold true. Let $\delta_{0}$ be defined in Lemma 7.2 (see (7.9) and (7.10)), and let $k>0$ be fixed.

Then there exists at least one solution of (5.1) such that

$$
\left\|w_{k}\right\|_{H_{0}^{1}(\Omega)} \leq Z_{\delta_{0}}
$$

where $Z_{\delta_{0}}$ is defined in Lemma 7.2 (see (7.9), (7.10) and (7.11)).

The proof of Theorem 5.1 consists in applying Schauder's fixed point theorem. First we prove the two following lemmas.

Lemma 5.2. Assume that (3.2), (3.3), (3.4), (3.5), (3.6) hold true. Let $k>0$ be fixed.

Then, for any $w \in H_{0}^{1}(\Omega)$, there exists a unique solution $W$ of the following semilinear problem

$$
\left\{\begin{array}{l}
W \in H_{0}^{1}(\Omega) \\
-\operatorname{div}(A(x) D W)+T_{k}\left(K_{\delta_{0}}(x, w, D w)\right) \operatorname{sign}_{k}(W) \\
=\left(1+\delta_{0}|w|\right) f(x)+a_{0}(x) w+a_{0}(x) g_{\delta_{0}}(w) \operatorname{sign}(w) \text { in } \mathcal{D}^{\prime}(\Omega) .
\end{array}\right.
$$

Moreover $W$ satisfies

$$
\left\{\begin{array}{l}
\alpha\|D W\|_{2}<\|f\|_{H^{-1}(\Omega)}+\frac{\delta_{0}\|f\|_{L^{1}(\log L)(\Omega)}}{2 \pi}\|D w\|_{2} \\
+\frac{C_{1}(q)\left\|a_{0}\right\|_{q}}{2 \pi}\|D w\|_{2}+\frac{\delta_{0} C_{2}(q)\left\|a_{0}\right\|_{q}}{(2 \pi)^{3 / 2}} \mid D w \|_{2}^{2} \quad \text { if } w \neq 0
\end{array}\right.
$$

where $C_{1}(q)$ and $C_{2}(q)$ are the constants given by (3.9). 
Proof. Problem (5.4) is of the form

$$
\left\{\begin{array}{l}
W \in H_{0}^{1}(\Omega), \\
-\operatorname{div}(A(x) D W)+\hat{b}(x) \operatorname{sign}_{k}(W)=\widehat{F}(x) \text { in } \mathcal{D}^{\prime}(\Omega),
\end{array}\right.
$$

where $\hat{b}(x)$ and $\hat{F}(x)$ are given. Since $\hat{b}(x)=T_{k}\left(K_{\delta_{0}}(x, w, D w)\right)$ belongs to $L^{\infty}(\Omega)$ and is nonnegative (see Remark 3.1 in [20]) and $\delta_{0} \geq \gamma$ (see (7.9)), since the function $\operatorname{sign}_{k}$ is continuous and nondecreasing, and since $\widehat{F}$ belong to $H^{-1}(\Omega)$ (see e.g. the computation which allows one to obtain (5.8) below), this problem has a unique solution.

Since $W \in H_{0}^{1}(\Omega)$, the use of $W$ as a test function in (5.4) is licit. Since the function $T_{k}\left(K_{\delta}(x, t, \zeta)\right)$ is nonnegative, this gives

$$
\left\{\begin{array}{l}
\int_{\Omega} A(x) D W D W d x \\
\leq \int_{\Omega}\left(1+\delta_{0}|w|\right) f(x) W d x \\
+\int_{\Omega} a_{0}(x) w W d x+\int_{\Omega} a_{0}(x) g_{\delta_{0}}(w) \operatorname{sign}(w) W d x
\end{array}\right.
$$

As in the computation made in Remark 4.4 to obtain the inequality (4.19), we use in (5.7) the coerciveness (3.3) of the matrix $A$, and classical properties about rearrangements. We obtain

$$
\left\{\begin{array}{l}
\alpha\|D W\|_{2}^{2} \leq\|f\|_{H^{-1}(\Omega)}\left\|D w_{\delta}\right\|_{2}\|D W\|_{2}+\frac{\delta_{0}\|f\|_{L^{1}(\log L)(\Omega)}}{2 \pi}\|D w\|_{2}\|D W\|_{2} \\
+\frac{C_{1}(q)\left\|a_{0}\right\|_{q}}{2 \pi}\|D w\|_{2}\|D W\|_{2}+\frac{C_{2}(q) \delta_{0}\left\|a_{0}\right\|_{q}}{(2 \pi)^{3 / 2}}\|D w\|_{2}^{2}\|D W\|_{2}
\end{array}\right.
$$

which implies (5.5).

Lemma 5.3. Assume (3.2), (3.3), (3.4), (3.5), (3.6) hold true. Assume moreover that the two smallness conditions (3.7) and (3.8) hold true. Let $k>0$ be fixed.

Let $w_{n}$ be a sequence such that

$$
w_{n} \rightarrow w \text { in } H_{0}^{1}(\Omega) \text { weakly and a.e. in } \Omega \text {. }
$$

Define $W_{n}$ as the unique solution of (5.4) for $w=w_{n}$, i.e.

$$
\left\{\begin{array}{l}
W_{n} \in H_{0}^{1}(\Omega), \\
-\operatorname{div}\left(A(x) D W_{n}\right)+T_{k}\left(K_{\delta_{0}}\left(x, w_{n}, D w_{n}\right)\right) \operatorname{sign}_{k}\left(W_{n}\right) \\
=\left(1+\delta_{0}\left|w_{n}\right|\right) f(x)+a_{0}(x) w_{n}+a_{0}(x) g_{\delta_{0}}\left(w_{n}\right) \operatorname{sign}\left(w_{n}\right) \quad \text { in } \quad \mathcal{D}^{\prime}(\Omega) .
\end{array}\right.
$$

Assume moreover that for a subsequence, still denoted by $n$, and for some $W^{\star} \in H_{0}^{1}(\Omega)$, $W_{n}$ satisfies

$$
W_{n} \rightarrow W^{\star} \text { in } H_{0}^{1}(\Omega) \text { weakly and a.e. in } \Omega \text {. }
$$

Then for the same subsequence one has

$$
W_{n} \rightarrow W^{\star} \quad \text { in } H_{0}^{1}(\Omega) \text { strongly. }
$$


Proof. Since $W_{n}-W^{\star} \in H_{0}^{1}(\Omega)$, the use of $\left(W_{n}-W^{\star}\right)$ as test function in (5.10) is licit. This gives

$$
\left\{\begin{array}{l}
\int_{\Omega} A(x) D\left(W_{n}-W^{\star}\right) D\left(W_{n}-W^{\star}\right) d x \\
=-\int_{\Omega} A(x) D W^{\star} D\left(W_{n}-W^{\star}\right) d x \\
-\int_{\Omega} T_{k}\left(K_{\delta_{0}}\left(x, w_{n}, D w_{n}\right)\right) \operatorname{sign}_{k}\left(W_{n}\right)\left(W_{n}-W^{\star}\right) d x \\
+\int_{\Omega}\left(1+\delta_{0}\left|w_{n}\right|\right) f(x)\left(W_{n}-W^{\star}\right) d x+\int_{\Omega} a_{0}(x) w_{n}\left(W_{n}-W^{\star}\right) d x \\
+\int_{\Omega} a_{0}(x) g_{\delta_{0}}\left(w_{n}\right) \operatorname{sign}\left(w_{n}\right)\left(W_{n}-W^{\star}\right) d x
\end{array}\right.
$$
infinity.

We claim that every term of the right-hand of (5.13) tends to zero as $n$ tends to

For the first term, we just use the fact that $W_{n}-W^{\star}$ tends to zero in $H_{0}^{1}(\Omega)$ weakly.

For the second term, we use the fact $T_{k}\left(K_{\delta_{0}}\left(x, w_{n}, D w_{n}\right)\right) \operatorname{sign}_{k}\left(W_{n}\right)$ is bounded in $L^{\infty}(\Omega)$, since $k$ is fixed, while $W_{n}-W^{\star}$ tends to zero in $L^{1}(\Omega)$ strongly.

For the last three terms we observe that, since $w_{n}$ and $W_{n}$ respectively converge almost everywhere to $w$ and to $W^{*}$ (see (5.9) and (5.11)), we have

$$
\begin{cases}\left(1+\delta_{0}\left|w_{n}\right|\right) f(x)\left(W_{n}-W^{\star}\right) \rightarrow 0 & \text { a.e. in } \Omega, \\ a_{0}(x) w_{n}\left(W_{n}-W^{\star}\right) \rightarrow 0 & \text { a.e. in } \Omega \\ a_{0}(x) g_{\delta_{0}}\left(w_{n}\right) \operatorname{sign}\left(w_{n}\right)\left(W_{n}-W^{\star}\right) \rightarrow 0 & \text { a.e. in } \Omega .\end{cases}
$$

We will now prove that each of the three sequences which appear in (5.14) are equiintegrable. Together with (5.14), this will imply that these sequences converge to zero in $L^{1}(\Omega)$ strongly, and this will prove that the three last terms of the right-hand side of (5.13) tend to zero as $n$ tends to infinity.

In order to prove that the sequence $\left(1+\delta_{0}\left|w_{n}\right|\right) f(x)\left(W_{n}-W^{\star}\right)$ is equiintegrable, we use the classical properties about rearrangements (2.1), (2.2) and (2.3). 
For any measurable set $E, E \subset \Omega$, we have

$$
\left\{\begin{array}{l}
\int_{E}\left|\left(1+\delta\left|w_{n}\right|\right) f(x)\left(W_{n}-W^{\star}\right)\right| d x \\
=\int_{E} f(x)\left(W_{n}-W^{\star}\right) d x+\delta \int_{E}\left|w_{n}\right| f(x)\left(W_{n}-W^{\star}\right) \mid d x \\
\leq \frac{\|f\|_{L^{1}(\Omega)}^{1 / 2}\|f\|_{L^{1}(\log L)(E)}^{1 / 2}}{(2 \pi)^{1 / 2}}\left\|D\left(W_{n}-W^{\star}\right)\right\|_{2}+\frac{\delta\|f\|_{L^{1}(\log L)(E)}}{2 \pi}\left\|D w_{n}\right\|_{2} \| D\left(W_{n}-W^{\star} \|_{2}\right. \\
\leq c_{1}\|f\|_{L^{1}(\log L)(E)}^{1 / 2}+c_{2}\|f\|_{L^{1}(\log L)(E)} .
\end{array}\right.
$$

where $c_{1}, c_{2}$ denote the constants which are independents of $n$.

Proving that the sequence $a_{0}(x) w_{n}\left(W_{n}-W^{\star}\right)$ is equiintegrable is similar, since for any measurable set $E, E \subset \Omega$, we have

$$
\left\{\begin{array}{l}
\int_{E}\left|a_{0}(x) w_{n}\left(W_{n}-W^{\star}\right)\right| d x \\
\leq\left(\int_{E}\left|a_{0}(x)\right|^{q} d x\right)^{1 / q} \frac{C_{1}(q)}{2 \pi}\left\|D w_{n}\right\|_{2}\left\|D\left(W_{n}-W^{\star}\right)\right\|_{2} \\
\leq c\left(\int_{E}\left|a_{0}(x)\right|^{q} d x\right)^{1 / q}
\end{array}\right.
$$

Finally, in order to prove that the sequence $a_{0}(x) g_{\delta_{0}}\left(w_{n}\right) \operatorname{sign}\left(w_{n}\right)\left(W_{n}-W^{\star}\right)$ is equiintegrable, we use as in (5.8), Hardy-Littlewood inequality; for any measurable set $E, E \subset \Omega$, we have

$$
\left\{\begin{array}{l}
\int_{E}\left|a_{0}(x) g_{\delta_{0}}\left(w_{n}\right) \operatorname{sign}\left(w_{n}\right)\left(W_{n}-W^{\star}\right)\right| d x \\
\leq \int_{E}\left|a_{0}(x)\right| \delta_{0}\left|w_{n}\right|^{2}\left|W_{n}-W^{\star}\right| d x \\
\leq\left(\int_{E}\left|a_{0}(x)\right|^{q} d x\right)^{1 / q} \delta_{0} \frac{C_{2}(q)}{(2 \pi)^{3 / 2}}\left\|D w_{n}\right\|_{2}^{2}\left\|D\left(W_{n}-W^{\star}\right)\right\|_{2} \leq c\left(\int_{E}\left|a_{0}(x)\right|^{q} d x\right)^{1 / q} .
\end{array}\right.
$$

We have proved that the right-hand side of (5.13) tends to zero. Since the matrix $A$ is coercive (see $(3.3)$ ), this proves that $W_{n}$ tends to $W^{\star}$ in $H_{0}^{1}(\Omega)$ strongly. Lemma 5.3 is proved.

Proof of Theorem 5.1. Recall that in this Theorem $k>0$ is fixed.

Consider the ball $B$ of $H_{0}^{1}(\Omega)$ defined by

$$
B=\left\{w \in H_{0}^{1}(\Omega):\|D w\|_{2} \leq Z_{\delta_{0}}\right\},
$$

where $Z_{\delta_{0}}$ is defined from $\delta_{0}$ by (7.11). 
Consider also the mapping $S: H_{0}^{1}(\Omega) \rightarrow H_{0}^{1}(\Omega)$ defined by

$$
S(w)=W,
$$

where for every $w \in H_{0}^{1}(\Omega), W$ is the unique solution of (5.4) (see Lemma 5.2).

We will apply Schauder's fixed point theorem in the Hilbert space $H_{0}^{1}(\Omega)$ to the mapping $S$ and to the ball $B$.

First step : In this step we prove that $S$ maps $B$ into itself.

Indeed by Lemma $5.2, W=S(w)$ satisfies (5.5); therefore, when $\|D w\|_{2} \leq Z_{\delta_{0}}$, one has, in view of the definition (7.7) the function $\Phi_{\delta}$ and of the property (7.10) of $Z_{\delta_{0}}$,

$$
\left\{\begin{aligned}
\alpha\|D W\|_{2} \leq & \|f\|_{H^{-1}(\Omega)}+\frac{\delta_{0}\|f\|_{L^{1}(\log L)(\Omega)}}{2 \pi}\|D w\|_{2} \\
& +\frac{C_{1}(q)\left\|a_{0}\right\|_{q}}{2 \pi}\|D w\|_{2}+\frac{\delta_{1} C_{2}(q)\left\|a_{0}\right\|_{q}}{(2 \pi)^{3 / 2}}\|D w\|_{2}^{2} \\
& \leq\|f\|_{H^{-1}(\Omega)}+\frac{\delta_{0}\|f\|_{L^{1}(\log L)(\Omega)}}{2 \pi} Z_{\delta_{0}} \\
& +\frac{C_{1}(q)\left\|a_{0}\right\|_{q}}{2 \pi} Z_{\delta_{0}}+\frac{\delta_{1} C_{2}(q)\left\|a_{0}\right\|_{q}}{(2 \pi)^{3 / 2}} Z_{\delta_{0}}^{2} \\
& =\alpha Z_{\delta_{0}}+\Phi_{\delta_{0}}\left(Z_{\delta_{0}}\right)=\alpha Z_{\delta_{0}}
\end{aligned}\right.
$$

i.e. $\|D W\|_{2} \leq Z_{\delta_{0}}$, or in other terms $W \in B$, which proves that $S(B) \subset B$.

Second step : In this step we prove that $S$ is continuous from $H_{0}^{1}(\Omega)$ strongly into $H_{0}^{1}(\Omega)$ strongly.

For this we consider a sequence such that

$$
w_{n} \in B, \quad w_{n} \rightarrow w \text { in } H_{0}^{1}(\Omega) \text { strongly, }
$$

and we define $W_{n}$ as $W_{n}=S\left(w_{n}\right)$, i.e. as the solution of (5.10).

The functions $w_{n}$ belong to $B$, and therefore the functions $W_{n}$ belong to $B$ in view of the first step. We can therefore extract a subsequence, still denoted by $n$, such that for some $W^{\star} \in H_{0}^{1}(\Omega)$,

$$
W_{n} \rightarrow W^{\star} \text { in } H_{0}^{1}(\Omega) \text { weakly and a.e. in } \Omega \text {. }
$$

We can moreover assume that for a further subsequence, still denoted by $n$,

$$
w_{n} \rightarrow w \text { a.e. in } \Omega \text { and } D w_{n} \rightarrow D w \text { a.e. in } \Omega \text {. }
$$

Since the assumptions of Lemma 5.3 are satisfied by the subsequences $w_{n}$ and $W_{n}$, the subsequence $W_{n}$ converges to $W^{\star}$ in $H_{0}^{1}(\Omega)$ strongly.

We now pass to the limit in equation (5.10) as $n$ tends to infinity by using the fact that $\operatorname{sign}_{k}(s)$ and $g_{\delta_{0}}(s) \operatorname{sign}(s)$ are Carathéodory functions, and the result of almost everywhere convergence(see Lemma 3.3 in [20]) as far as $T_{k}\left(K_{\delta_{0}}\left(x, w_{n}, D w_{n}\right)\right)$ is concerned (this point is the only point of the proof of Theorem 5.1 where the assumption 
of strong $H_{0}^{1}(\Omega)$ convergence in (5.18), or more exactly its consequence (5.20), is used). This implies that $W^{\star}$ is a solution of (5.4). Since the solution of (5.4) is unique, one has $W^{\star}=S(w)$.

In view of the fact that $W^{\star}$ is uniquely determined, we conclude that it was not necessary to extract a subsequence in (5.19) and (5.20), and that the whole sequence $W_{n}=S\left(w_{n}\right)$ converges in $H_{0}^{1}(\Omega)$ strongly to $W^{\star}=S(w)$. This proves the continuity of the application $S$.

Third step : In this step we prove that $S(B)$ is precompact in $H_{0}^{1}(\Omega)$.

For this we consider a sequence $w_{n} \in B$ and we define $W_{n}$ as $W_{n}=S\left(w_{n}\right)$; in other terms $W_{n}$ is the solution of (5.10). Since $w_{n}$ and $W_{n}$ belong to $B$, they are bounded in $H_{0}^{1}(\Omega)$, and we can extract a subsequence, still denoted by $n$, such that

$$
\begin{gathered}
w_{n} \rightarrow w \quad \text { in } H_{0}^{1}(\Omega) \text { weakly and a.e. in } \Omega, \\
W_{n} \rightarrow W^{\star} \quad \text { in } H_{0}^{1}(\Omega) \text { weakly and a.e. in } \Omega .
\end{gathered}
$$

Since $w_{n}$ and $W_{n}$ satisfies the assumptions of Lemma 5.3, we have

$$
W_{n} \rightarrow W^{\star} \quad \text { in } H_{0}^{1}(\Omega) \text { strongly. }
$$

This proves that $S(B)$ is precompact in $H_{0}^{1}(\Omega)$ (note that in contrast with the second step, we do not need here to prove that $\left.W^{\star}=S(w)\right)$.

End of the proof of Theorem 5.1 We have proved that the application $S$ and the ball $B$ satisfy the assumptions of Schauder's fixed point theorem. Therefore there exists at least one $w_{k} \in B$ such that $S\left(w_{k}\right)=w_{k}$. This proves Theorem 5.1.

\section{Proof of Theorem 4.3}

Theorem 5.1 asserts that for for every $k>0$ fixed there exists at least a solution $w_{k}$ of (5.1) which satisfies (5.3). We can therefore extract a subsequence, still denoted by $k$, such that for some $w^{\star} \in H_{0}^{1}(\Omega)$

$$
w_{k} \rightarrow w^{\star} \quad \text { in } H_{0}^{1}(\Omega) \text { weakly and a.e. in } \Omega,
$$

where $w^{\star}$ satisfies

$$
\left\|w^{\star}\right\|_{H_{0}^{1}(\Omega)} \leq Z_{\delta_{0}}
$$

i.e. (4.14).

In this Section we will first prove that for this subsequence

$$
w_{k} \rightarrow w^{\star} \text { in } H_{0}^{1}(\Omega) \text { strongly, }
$$

and then that $w^{\star}$ is a solution of (4.13) (which satisfies (4.14)). This will prove Theorem 4.3.

To prove (6.3), we use a technique which traces back to [7] (see also [15]). 
For $n>0$, we define $G_{n}: \mathbb{R} \rightarrow \mathbb{R}$ as the remainder of the truncation at height $n$, namely

$$
G_{n}(s)=s-T_{n}(s), \quad \forall s \in \mathbb{R}
$$

where $T_{n}$ is the truncation at height $n$ defined by (4.15), or in other terms

$$
G_{n}(s)= \begin{cases}s+n & \text { if } \quad s \leq-n, \\ 0 & \text { if }-n \leq s \leq n, \\ s-n & \text { if } s \geq n\end{cases}
$$

First we use the two following Lemmas.

Lemma 6.1. [15] Assume that (3.2), (3.3), (3.4), (3.5), (3.6) hold true. Assume moreover that the two smallness conditions (3.7) and (3.8) hold true. Let $w_{k}$ be a solution of (5.1). Assume finally that the subsequence $w_{k}$ satisfies (6.1).

Then for this subsequence we have

$$
\limsup _{k \rightarrow+\infty} \int_{\Omega}\left|D G_{n}\left(w_{k}\right)\right|^{2} d x \rightarrow 0 \text { as } n \rightarrow+\infty
$$

Lemma 6.2. [15] Assume that (3.2), (3.3), (3.4), (3.5), (3.6) hold true. Assume moreover that the two smallness conditions (3.7) and (3.8) hold true. Let $w_{k}$ be a solution of (5.1). Assume finally that the subsequence $w_{k}$ satisfies (6.1).

Then for this subsequence we have for every $n>0$ fixed

$$
T_{n}\left(w_{k}\right) \rightarrow T_{n}\left(w^{\star}\right) \text { in } H_{0}^{1}(\Omega) \text { strongly as } k \rightarrow+\infty \text {. }
$$

\section{End of the proof of Theorem 4.3}

First step Since we have

$$
w_{k}-w^{\star}=T_{n}\left(w_{k}\right)+G_{n}\left(w_{k}\right)-T_{n}\left(w^{\star}\right)-G_{n}\left(w^{\star}\right),
$$

and since by Lemma 6.2 (see (6.7)) we have

$$
\left\|T_{n}\left(w_{k}\right)-T_{n}\left(w^{\star}\right)\right\|_{H_{0}^{1}(\Omega)} \rightarrow 0 \quad \text { as } \quad k \rightarrow+\infty \text { for every } n>0 \text { fixed, }
$$

while by Lemma 6.1 (see (6.6)) we have

$$
\limsup _{n \rightarrow+\infty} \limsup _{k \rightarrow+\infty}\left\|G_{n}\left(w_{k}\right)\right\|_{H_{0}^{1}(\Omega)}=0
$$

and while we have

$$
\limsup _{n \rightarrow+\infty}\left\|G_{n}\left(w^{\star}\right)\right\|_{H_{0}^{1}(\Omega)}=0,
$$

since $w^{\star} \in H_{0}^{1}(\Omega)$, we conclude that

$$
w_{k} \rightarrow w^{\star} \text { in } H_{0}^{1}(\Omega) \text { strongly as } k \rightarrow+\infty,
$$

which is nothing but (6.3).

Second step Let us now pass to the limit in (5.1) as $k$ tends to infinity. This is easy for the first term of the left-hand side of (5.1) as well as for the three terms of the right-hand 
side of (5.1), which is similar to the one that we used in the proof of Lemma 5.3.

It remains to pass to the limit in the second term of the left-hand side of (5.1), namely in

$$
T_{k}\left(K_{\delta_{0}}\left(x, w_{k}, D w_{k}\right)\right) \operatorname{sign}_{k}\left(w_{k}\right)
$$

We first observe that $K_{\delta}(x, s, \zeta)>0$ and $\delta_{0} \geq \gamma$ (see (7.9)), we have

$$
\left\{\begin{array}{l}
\left|T_{k}\left(K_{\delta_{0}}\left(x, w_{k}, D w_{k}\right)\right) \operatorname{sign}_{k}\left(w_{k}\right)\right| \leq\left|K_{\delta_{0}}\left(x, w_{k}, D w_{k}\right)\right| \\
\leq\left(c_{0}+\delta_{0}\right)\|A\|_{\infty}\left|D w_{k}\right|^{2} \quad \text { a.e. in } \Omega
\end{array}\right.
$$

which implies that the functions $T_{k}\left(K_{\delta_{0}}\left(x, w_{k}, D w_{k}\right)\right) \operatorname{sign}_{k}\left(w_{k}\right)$ are equiintegrable since $D w_{k}$ converges strongly to $D w^{\star}$ in $L^{2}(\Omega)^{2}$.

Extracting if necessary a subsequence, still denoted by $k$, such that

$$
D w_{k} \rightarrow D w^{\star} \text { a.e. in } \Omega \text {, }
$$

we claim that

$$
T_{k}\left(K_{\delta_{0}}\left(x, w_{k}, D w_{k}\right)\right) \operatorname{sign}_{k}\left(w_{k}\right) \rightarrow K_{\delta_{0}}\left(x, w^{\star}, D w^{\star}\right) \operatorname{sign}\left(w^{\star}\right) \quad \text { a.e. in } \Omega \text {. }
$$

On the first hand we use the following almost everywhere convergence, which asserts that

and the fact that for every $s \in \mathbb{R}$

$$
K_{\delta_{0}}\left(x, w_{k}, D w_{k}\right) \rightarrow K_{\delta_{0}}\left(x, w^{\star}, D w^{\star}\right) \text { a.e. in } \Omega,
$$

$$
T_{k}\left(s_{k}\right) \rightarrow s \quad \text { if } k \rightarrow+\infty \quad \text { when } \quad s_{k} \rightarrow s
$$

to deduce that

$$
T_{k}\left(K_{\delta_{0}}\left(x, w_{k}, D w_{k}\right)\right) \rightarrow K_{\delta_{0}}\left(x, w^{\star}, D w^{\star}\right) \text { a.e. in } \Omega .
$$

On the other hand we use the fact that

$$
\operatorname{sign}_{k}\left(w_{k}\right) \rightarrow \operatorname{sign}\left(w^{\star}\right) \text { a.e. in }\left\{y \in \Omega: w^{\star}(y) \neq 0\right\},
$$

which together with (6.10) proves convergence (6.9) in the set $\left\{y \in \Omega: w^{\star}(y) \neq 0\right\}$.

Finally, as far as convergence in the set $\left\{y \in \Omega: w^{\star}(y)=0\right\}$ is concerned, convergence (6.10), the fact that

$$
K_{\delta_{0}}\left(x, w^{\star}, D w^{\star}\right)=0 \text { a.e. in }\left\{y \in \Omega: w^{\star}(y)=0\right\},
$$

and the fact that $\left|\operatorname{sign}_{k}(s)\right| \leq 1$ for every $s \in \mathbb{R}$ together prove that

$$
\left\{\begin{array}{l}
T_{k}\left(K_{\delta_{0}}\left(x, w_{k}, D w_{k}\right)\right) \operatorname{sign}_{k}\left(w_{k}\right) \rightarrow 0=K_{\delta_{0}}\left(x, w^{\star}, D w^{\star}\right) \operatorname{sign}\left(w^{\star}\right) \\
\text { a.e. in }\left\{y \in \Omega: w^{\star}(y)=0\right\} .
\end{array}\right.
$$

This completes the proof of (6.9).

The equiintegrability and the almost everywhere convergence of the functions $T_{k}\left(K_{\delta_{0}}\left(x, w_{k}, D w_{k}\right)\right) \operatorname{sign}_{k}\left(w_{k}\right)$ then imply that

$T_{k}\left(K_{\delta_{0}}\left(x, w_{k}, D w_{k}\right)\right) \operatorname{sign}_{k}\left(w_{k}\right) \rightarrow K_{\delta_{0}}\left(x, w^{\star}, D w^{\star}\right) \operatorname{sign}\left(w^{\star}\right) \quad$ in $\quad L^{1}(\Omega)$ strongly.

This proves that $w^{\star}$ satisfies (4.13). Since $w^{\star}$ also satisfies (4.14) (see (6.2)), Theorem 4.3 is proved. 


\section{Appendix}

In this Appendix, we give an estimate of the function $g_{\delta}$ defined by (4.6) (see Lemma 7.1), and the definitions of the constants $\delta_{0}$ and $Z_{\delta_{0}}$ which appear in Theorem 3.1 (see Lemma 7.2).

\subsection{An estimate for the function $\mathrm{g}_{\delta}$.}

Lemma 7.1. For $\delta>0$, let $g_{\delta}: \mathbb{R} \rightarrow \mathbb{R}$ be the function defined by (4.6), i.e. by

$$
g_{\delta}(t)=-|t|+\frac{1}{\delta}(1+\delta|t|) \log (1+\delta|t|), \quad \forall t \in \mathbb{R} .
$$

Then

$$
0 \leq g_{\delta}(t) \leq \delta|t|^{2}, \quad \forall t \in \mathbb{R}, \quad \forall \delta, 0<\delta \leq \delta_{1} .
$$

Proof. Let $g: \mathbb{R}^{+} \rightarrow \mathbb{R}$ be the function defined by

$$
g(\tau)=-\tau+(1+\tau) \log (1+\tau), \quad \forall \tau \geq 0 .
$$

Since $g(0)=0$ and $g^{\prime}(\tau) \geq 0$, one has

$$
g(\tau) \geq 0, \quad \forall \tau \geq 0 .
$$

Since $\log (1+\tau)<\tau$ for $\tau>0$, one has

$$
g(\tau)<\tau^{2}, \quad \forall \tau, \tau>0
$$

Since

$$
g_{\delta}(t)=\frac{1}{\delta} g(\delta|t|), \quad \forall t \in \mathbb{R},
$$

one deduces from (7.4) that $g_{\delta}$ satisfies (7.2).

7.2. Definition of $\boldsymbol{\delta}_{\mathbf{0}}$ and $Z_{\boldsymbol{\delta}_{0}}$. The goal of this Subsection is to define the constants $\delta_{0}$ and $Z_{\delta_{0}}$ which appear in Theorem 3.1. We will prove the following result.

Lemma 7.2. Assume that (3.2), (3.3), (3.4), (3.5) and (3.6) hold true. Assume moreover that the two smallness conditions (3.7) and (3.8) hold true.

Let $\delta_{1}$ be the number defined by

$$
\delta_{1}=\frac{2 \pi \alpha-C_{1}(q)\left\|a_{0}\right\|_{q}}{\|f\|_{L^{1}(\log L)(\Omega)}} .
$$

One has

$$
\delta_{1}>\gamma
$$

For $\delta \geq 0$, let $\Phi_{\delta}: \mathbb{R}^{+} \rightarrow \mathbb{R}$ (see Fig. 2) be the function defined by

$$
\begin{aligned}
\Phi_{\delta}(X)= & \delta_{1} C_{2}(q)\left\|a_{0}\right\|_{q} X^{2} \\
& -\frac{2 \pi \alpha-C_{1}(q)\|a\|_{q}-\delta\|f\|_{L^{1}(\log L)(\Omega)}}{2 \pi} X+\|f\|_{H^{-1}(\Omega)},
\end{aligned}
$$


where $C_{1}(q)$ and $C_{2}(q)$ are the constants given in (3.9).

Then, for $0 \leq \delta \leq \delta_{1}$, the function $\Phi_{\delta}$ has a unique minimizer $Z_{\delta}$ on $\mathbb{R}^{+}$, which is given by

$$
Z_{\delta}=\frac{2 \pi \alpha-C_{1}(q)\left\|a_{0}\right\|_{q}-\delta\|f\|_{L^{1}(\log L)(\Omega)}}{4 \pi \delta_{1} C_{2}(q)\left\|a_{0}\right\|_{q}}, \quad \text { for } \quad 0 \leq \delta \leq \delta_{1} .
$$

Moreover, there exists a unique number $\delta_{0}$ such that

$$
\gamma \leq \delta_{0}<\delta_{1}
$$

and

$$
\Phi_{\delta_{0}}\left(Z_{\delta_{0}}\right)=0
$$

This number is the number $\delta_{0}$ which appear in Theorem 3.1, and $Z_{\delta_{0}}$ is then defined from $\delta_{0}$ through formula (7.8), namely by

$$
Z_{\delta_{0}}=\frac{2 \pi \alpha-C_{1}(q)\left\|a_{0}\right\|_{q}-\delta_{0}\|f\|_{L^{1}(\log L)(\Omega)}}{4 \pi \delta_{1} C_{2}(q)\left\|a_{0}\right\|_{q}}
$$

Remark 7.3. Let us explain the meaning of the results stated in Lemma 7.2.

As we will see in the proof of Lemma 7.2 (see also Fig. 2), the function $\Phi_{\delta}$ is the restriction to $\mathbb{R}^{+}$of a function which looks like a convex parabola. This function attains its minimum at a unique point $Z_{\delta}$, and for $\delta$ which satisfies $\delta<\delta_{1}$ with $\delta_{1}$ given by (7.5), one has $Z_{\delta}>0$.

The smallness condition (3.7) is equivalent to the fact that $\delta_{1}>\gamma$, and the smallness condition (3.8) to the fact that the minimum $\Phi_{\gamma}\left(Z_{\gamma}\right)$ of $\Phi_{\gamma}$ is nonpositive. For $\delta=\delta_{1}$, the minimum $\Phi_{\delta_{1}}\left(Z_{\delta_{1}}\right)$ of $\Phi_{\delta_{1}}$ is equal to $\|f\|_{H^{-1}(\Omega)}$, which is strictly positive. Therefore it can be proved that there exists some $\delta_{0}$ with $\gamma \leq \delta_{0}<\delta_{1}$ (see (7.9)) such that the minimum $\Phi_{\delta_{0}}\left(Z_{\delta_{0}}\right)$ of $\Phi_{\delta_{0}}$ is equal to zero (see (7.10)), or in other terms such that the function $\Phi_{\delta_{0}}$ has a double zero in $Z_{\delta_{0}}$. Moreover, when $\gamma<\delta_{0}$, for every $\delta$ with $\gamma \leq \delta<\delta_{0}$, the function $\Phi_{\delta}$ has two distinct zeros $Y_{\delta}^{-}$and $Y_{\delta}^{+}$with $Y_{\delta}^{-}<Y_{\delta}^{+}$which satisfy $0<Y_{\delta}^{-}<Z_{\delta_{0}}<Y_{\delta}^{+}$

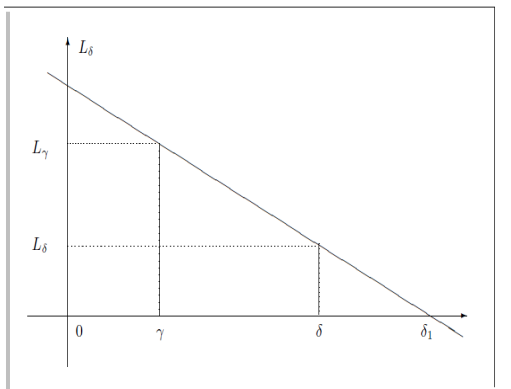

Fig. 1: The graph of the straight line $L_{\delta}$

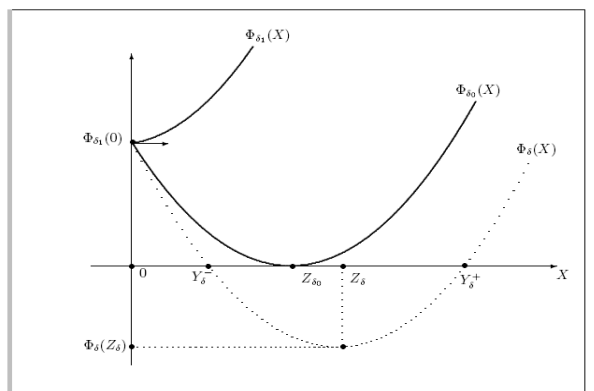

Fig. 2: The graphs of the functions $\Phi_{\delta}(X)$ 
Proof of Lemma 7.2 For $\delta \geq 0$, let $L_{\delta}$ be the constant defined by (see Fig. 1)

$$
L_{\delta}=\frac{1}{2 \pi}\left(2 \pi \alpha-C_{1}(q)\left\|a_{0}\right\|_{q}-\delta\|f\|_{L^{1}(\log L)(\Omega)}\right),
$$

where $C_{1}(q)$ and $C_{2}(q)$ are two positives constants satisfy (3.9). Note that $L_{\delta}$ is decreasing with respect to $\delta$.

Since $\delta_{1}$ is defined by (7.5), one has $L_{\delta_{1}}=0$. On the other hand, the first smallness condition (3.7) is nothing but $L_{\gamma}>0$. Since $L_{\delta}$ is decreasing in $\delta$, one has $\delta_{1}>\gamma$, i.e. (7.6).

Let us now study the family of functions $\Phi_{\delta}: \mathbb{R}^{+} \rightarrow \mathbb{R}$ defined by (7.7), i.e., in view of the definition (7.12) of $L_{\delta}$, by

$$
\Phi_{\delta}(X)=\delta_{1} C_{2}(q)\left\|a_{0}\right\|_{q} X^{2}-L_{\delta} X+\|f\|_{H^{-1}(\Omega)}, \quad \forall X \geq 0,
$$

(see Fig. 2).

Since $a_{0} \neq 0$ (see (3.6)), each function $\Phi_{\delta}$ looks like the restriction to $\mathbb{R}^{+}$of a convex parabola. When $0 \leq \delta \leq \delta_{1}$, one has $L_{\delta} \geq 0$, and this convex parabola has a unique minimizer $Z_{\delta}$ on $\mathbb{R}^{+}$which is also the minimizer of the function $\Phi_{\delta}$. A simple computation shows that $Z_{\delta}$ is given by

$$
Z_{\delta}=\frac{L_{\delta}}{2 \delta_{1} C_{2}(q)\left\|a_{0}\right\|_{q}}=\frac{2 \pi \alpha-C_{1}(q)\left\|a_{0}\right\|_{q}-\delta_{0}\|f\|_{L^{1}(\log L)(\Omega)}}{4 \pi \delta_{1} C_{2}(q)\left\|a_{0}\right\|_{q}}
$$

i.e (7.8), and that the minimum of $\Phi_{\delta}$, namely $\Phi_{\delta}\left(Z_{\delta}\right)$, is given by

$$
\begin{aligned}
\Phi_{\delta}\left(Z_{\delta}\right) & =\|f\|_{H^{-1}(\Omega)}-\frac{L_{\delta}^{2}}{4 \delta_{1} C_{2}(q)\left\|a_{0}\right\|_{q}} \\
& =\|f\|_{H^{-1}(\Omega)}-\frac{\left(2 \pi \alpha-C_{1}(q)\left\|a_{0}\right\|_{q}-\delta\|f\|_{L^{1}(\log L)(\Omega)}\right)^{2}}{16 \pi^{2} \delta_{1} C_{2}(q)\left\|a_{0}\right\|_{q}} .
\end{aligned}
$$

When $0 \leq \delta \leq \delta_{1}$, the function $L_{\delta}$ is nonnegative, continuous and decreasing with respect to $\delta$. Therefore $Z_{\delta}$ is continuous and decreasing with respect to $\delta$, while $\Phi_{\delta}\left(Z_{\delta}\right)$ is continuous and increasing with respect to $\delta$.

When $\delta=\delta_{1}$, one has $L_{\delta_{1}}=0$, the function $\Phi_{\delta_{1}}$ attains its minimum in $Z_{\delta_{1}}=0$, and $\Phi_{\delta_{1}}\left(Z_{\delta_{1}}\right)=\|f\|_{H^{-1}(\Omega)}$, while the second smallness condition (3.8) is nothing but $\Phi_{\gamma}\left(Z_{\gamma}\right) \leq 0$. Therefore there exists a unique $\delta_{0}$ with $\gamma \leq \delta_{0}<\delta_{1}$ such that $\Phi_{\delta_{0}}\left(Z_{\delta_{0}}\right)=0$. This is the definition of $\delta_{0}$ given by (7.9) and (7.10) in Lemma 7.2.

Lemma 7.2 is proved.

\section{Acknowledgments}

This work was carried out during the author's visits to the Laboratoire Jacques-Louis Lions, Sorbonne Université Campus Pierre et Marie Curie (Paris VI) and would like to thank Professor F. Murat for his hospitality. 


\section{References}

[1] A. Alvino, P.-L. Lions, G. Trombetti, Comparison results for elliptic and parabolic equations via Schwarz symmetrization, Ann. Inst. H. Poincaré Anal. non linéaire 7 (1990), 37-65.

[2] A. Alvino, Un caso limite della disuguaglianza di Sobolev in spazi di Lorentz, Rend. Acc. Sci. Fis. Mat. Napoli, 44 (1977), 105-112.

[3] D. Arcoya, C. De Coster, L. Jeanjean, K. Tanaka, Continuum of solutions for an elliptic problem with critical growth in the gradient, J. Funct. Anal. 268 (2015), 2298-2335.

[4] G. Barles, A.-P. Blanc, C. Georgelin, M. Kobylanski, Remarks on the maximum principle for nonlinear elliptic PDEs with quadratic growth conditions, Ann. Sc. Norm. Sup. Pisa 28 (1999), 381-404.

[5] G. Barles, F. Murat, Uniqueness and the maximum principle for quasilinear elliptic equations with quadratic growth conditions, Arch. Rational. Mech. Anal. 133 (1995), $77-101$.

[6] G. Barles, A. Porretta, Uniqueness for unbounded solutions to stationary viscous Hamilton-Jacobi equations, Ann. Sc. Norm. Sup. Pisa 5 (2006), 107-136.

[7] A. Bensoussan, L. Boccardo, F. Murat, On a nonlinear partial differential equation having natural growth terms and unbounded solution, Ann. Inst. H. Poincaré Anal. non linéaire 5 (1988), 347-364.

[8] L. Boccardo, F. Murat, J.-P. Puel, Existence de solutions non bornées pour certaines équations quasi-linéaires, Portugaliæ Math. 41 (1982), 507-534.

[9] L. Boccardo, F. Murat, J.-P. Puel, Existence de solutions faibles pour des équations elliptiques quasi-linéaires à croissance quadratique, in Nonlinear Partial Differential Equations and their Applications, Collège de France Seminar Vol. IV (H. Brezis and J.-L. Lions eds.), Research Notes in Math. 84 (1983), Pitman, London, 19-73.

[10] L. Boccardo, F. Murat, J.-P. Puel, Résultats d'existence pour certains problèmes elliptiques quasi-linéaires, Ann. Sc. Norm. Sup. Pisa 11 (1984), 213-235.

[11] L. Boccardo, F. Murat, J.-P. Puel, Existence of bounded solutions for nonlinear elliptic unilateral problems, Ann. Mat. Pura App. 152 (1988), 183-196.

[12] L. Boccardo, F. Murat, J.-P. Puel, $L^{\infty}$ estimate for some nonlinear elliptic partial differential equations and application to an existence result, SIAM J. Math. Anal. 23 (1992), 326-333.

[13] F. Chiacchio, An existence result for a nonlinear problem in a limit case, Mathematiche, Vol. 53, 2(1998), 293-304.

[14] A. Dall'Aglio, D. Giachetti, J.-P. Puel, Nonlinear elliptic equations with natural growth in general domains, Ann. Mat. Pura Appl. 181 (2002), 407-426.

[15] V. Ferone, F. Murat, Quasilinear problems having quadratic growth in the gradient: an existence result when the source term is small, in Equations aux dérivées partielles et applications, Articles dédiés à Jacques-Louis Lions, (1988), Gauthiers-Villars, Paris, 497-515. 
[16] V. Ferone, F. Murat, Nonlinear problems having natural growth in the gradient: an existence result when the source terms are small, Nonlinear Anal. 42 (2000), 13091326.

[17] V. Ferone, F. Murat, Nonlinear elliptic equations in the gradient and source terms in Lorentz spaces, J. Diff. Eq. 256 (2014), 577-608.

[18] V. Ferone, M.-R. Posteraro, On a class of quasilinear elliptic equations with quadratic growth in the gradient, Nonlinear Anal. Th. Math. Appl. 20 (1993), 703-711.

[19] N. Grenon-Isselkou, J. Mossino, Existence de solutions bornées pour certaines équations elliptiques quasilinéaires, C. R. Math. Acad. Sci. Paris 321 (1995), 5156.

[20] B. Hamour, F. Murat, Quasilinear problems involving a perturbation with quadratic growth in the gradient and a noncoercive zeroth order term, Rend. Lincei Mat. Appl. 27 (2016), 195-233

[21] B. Hamour, F. Murat, Nonlinear problems involving a perturbation with natural growth in the gradient and a noncoercive zeroth order term, in preparation.

[22] L. Jeanjean, B. Sirakov, Existence and multiplicity for elliptic problems with quadratic growth in the gradient, Comm. Part. Diff. Eq. 38 (2013), 244-264.

[23] C. Maderna, C. Pagani, S. Salsa, Quasilinear elliptic equations with quadratic growth in the gradient, J. Diff. Eq. 97 (1992), 54-70.

[24] J. Mossino, Inégalités isopérimétriques et applications en physique, Hermann, Paris, (1984).

[25] A. Porretta, The ergodic limit for a viscous Hamilton Jacobi equation with Dirichlet conditions, Rend. Lincei Mat. Appl. 21 (2010), 59-78.

[26] J.-M. Rakotoson, Réearrangement relatif dans les équations elliptiques quasilinéaires avec un second membre distribution: application à un théorème d'existence et de régularité, J. Diff. Eq. 66 (1987), 391-419.

[27] P. Souplet, A priori estimates and bifurcation of solutions for an elliptic equation with semidefinite critical growth in the gradient, Nonlinear Anal. 121 (2015), 412423.

\section{Received}

Boussad Hamour,

Laboratoire Equations aux dérivées partielles non linéaires

et histoire des mathématiques,

Ecole Normale Supérieure B. Ibrahimi,

Boîte Postale 92, Vieux Kouba

16050 Alger, Algérie

E-mail: hamour@ens-kouba.dz 